\title{
BMJ
}

\section{Calcium supplements with or without vitamin D and risk of cardiovascular events: reanalysis of the Women's Health Initiative limited access dataset and meta-analysis}

\author{
Mark J Bolland, senior research fellow, ${ }^{1}$ Andrew Grey, associate professor, ${ }^{1}$ Alison Avenell, clinical research \\ fellow, ${ }^{2}$ Greg D Gamble, research fellow, ${ }^{1}$ lan R Reid, professor of medicine and endocrinology ${ }^{1}$
}

${ }^{1}$ Department of Medicine, University of Auckland, Private Bag 92 019, Auckland 1142, New Zealand

${ }^{2}$ Health Services Research Unit, University of Aberdeen,

Foresterhill, Aberdeen AB25 2ZD, Scotland

Correspondence to: I R Reid i.reid@auckland.ac.nz

Cite this as: $B M J$ 2011;342:d2040 doi:10.1136/bmj.d2040

\section{ABSTRACT}

Objectives To investigate the effects of personal calcium supplement use on cardiovascular risk in the Women's Health Initiative Calcium/Vitamin D Supplementation Study (WHI CaD Study), using the WHI dataset, and to update the recent meta-analysis of calcium supplements and cardiovascular risk.

Design Reanalysis of WHI CaD Study limited access dataset and incorporation in meta-analysis with eight other studies.

Data source WHI CaD Study, a seven year, randomised, placebo controlled trial of calcium and vitamin D ( $1 \mathrm{~g}$ calcium and $400 \mathrm{IU}$ vitamin D daily) in 36282 community dwelling postmenopausal women.

Main outcome measures Incidence of four cardiovascular events and their combinations (myocardial infarction, coronary revascularisation, death from coronary heart disease, and stroke) assessed with patient-level data and trial-level data.

Results In the WHI CaD Study there was an interaction between personal use of calcium supplements and allocated calcium and vitamin D for cardiovascular events. In the 16718 women (46\%) who were not taking personal calcium supplements at randomisation the hazard ratios for cardiovascular events with calcium and vitamin $D$ ranged from 1.13 to $1.22(P=0.05$ for clinical myocardial infarction or stroke, $\mathrm{P}=0.04$ for clinical myocardial infarction or revascularisation), whereas in the women taking personal calcium supplements cardiovascular risk did not alter with allocation to calcium and vitamin D. In meta-analyses of three placebo controlled trials, calcium and vitamin D increased the risk of myocardial infarction (relative risk 1.21 ( $95 \%$ confidence interval 1.01 to 1.44$), \mathrm{P}=0.04)$, stroke (1.20 (1.00 to 1.43$), P=0.05)$, and the composite of myocardial infarction or stroke (1.16 (1.02 to 1.32$), \mathrm{P}=0.02)$. In metaanalyses of placebo controlled trials of calcium or calcium and vitamin D, complete trial-level data were available for 28072 participants from eight trials of calcium supplements and the WHI CaD participants not taking personal calcium supplements. In total 1384 individuals had an incident myocardial infarction or stroke. Calcium or calcium and vitamin D increased the risk of myocardial infarction (relative risk 1.24 (1.07 to 1.45$), P=0.004)$ and the composite of myocardial infarction or stroke (1.15 (1.03 to 1.27), $\mathrm{P}=0.009$ ).

Conclusions Calcium supplements with or without vitamin D modestly increase the risk of cardiovascular events, especially myocardial infarction, a finding obscured in the WHI CaD Study by the widespread use of personal calcium supplements. A reassessment of the role of calcium supplements in osteoporosis management is warranted.

\section{INTRODUCTION}

Calcium supplements, with or without vitamin D, are widely used for the prevention and treatment of osteoporosis. Recently, we reported increases in rates of cardiovascular events in women allocated to calcium supplements in the Auckland Calcium Study, a five year randomised, placebo controlled trial in healthy older women, in which cardiovascular events were pre-specified secondary end points. ${ }^{1}$ Subsequently, we carried out a meta-analysis of cardiovascular events in randomised, placebo controlled trials of calcium supplements (without vitamin D co-administration). ${ }^{2}$ Calcium supplements significantly increased the risk of myocardial infarction by $31 \%$ in five trials involving 8151 participants where patient level data were available, and by $27 \%$ in 11 trials involving 11921 participants where trial level data were available. ${ }^{2}$ There were no statistically significant increases in the risk of stroke; the composite end point of myocardial infarction, stroke, or sudden death; or death (relative risks or hazard ratios ranged from 1.07 to 1.20 ), but the metaanalyses did not have sufficient power to detect effect sizes of these magnitudes. ${ }^{2}$

An important question that arises is whether coadministered calcium and vitamin $\mathrm{D}$ affects cardiovascular risk. The Women's Health Initiative reported no adverse effect of calcium and vitamin D $(1 \mathrm{~g}$ calcium/400 IU vitamin D daily) on any cardiovascular end point in their large $(\mathrm{n}=36282)$, seven year, randomised, placebo controlled trial. ${ }^{34}$ However, $54 \%$ of the participants were taking personal (non-protocol) 
calcium supplements at randomisation and $47 \%$ were taking personal vitamin D supplements, effectively rendering this trial a comparison of higher dose and lower dose calcium and vitamin D for most of the participants.

Allowing clinical trial participants free access to the intervention being studied is unusual and has the potential to obscure both adverse and beneficial effects. We hypothesised that the frequent personal use of calcium supplements might have obscured an adverse effect of calcium and vitamin $\mathrm{D}$ on cardiovascular risk. Therefore, we analysed the limited access dataset of the Women's Health Initiative Calcium/ Vitamin D Supplementation Study (WHI CaD Study) to determine whether there were interactions between personal use of calcium supplements and allocation to calcium and vitamin D supplementation for cardiovascular events. Using data from women not using personal calcium supplements at randomisation in the study, we have updated our previous meta-analysis to provide the best current estimate of the effects of calcium supplements, with or without vitamin D, on the risk of cardiovascular events.

Table 1/Characteristics of participants in the WHI CaD Study at randomisation, grouped by personal use of calcium supplements. Values are percentages (numbers) unless stated otherwise

\begin{tabular}{|c|c|c|c|c|}
\hline \multirow[b]{2}{*}{ Characteristic } & \multicolumn{2}{|c|}{ No personal use of calcium } & \multicolumn{2}{|c|}{ Any personal use of calcium } \\
\hline & $\begin{array}{c}\mathrm{CaD} \\
(\mathrm{n}=8429)\end{array}$ & $\begin{array}{l}\text { Placebo } \\
(\mathrm{n}=8289)\end{array}$ & $\begin{array}{c}\mathrm{CaD} \\
(\mathrm{n}=9747)\end{array}$ & $\begin{array}{l}\text { Placebo } \\
(\mathrm{n}=9817)\end{array}$ \\
\hline \multicolumn{5}{|l|}{ Age (years): } \\
\hline Mean (SD) & $62.9(7.0)$ & $62.9(7.0)$ & $63.9(6.9)$ & $63.9(6.8)$ \\
\hline $50-60$ & 39 (3259) & 38 (3148) & $31(3030)$ & $32(3100)$ \\
\hline $60-70$ & $43(3633)$ & $44(3625)$ & 49 (4741) & $48(4726)$ \\
\hline$\geq 70$ & $18(1537)$ & $18(1516)$ & 20 (1976) & 20 (1991) \\
\hline \multicolumn{5}{|l|}{ Body mass index $\left(\mathrm{kg} / \mathrm{m}^{2}\right)$ : } \\
\hline Mean (SD) & $29.4(5.9)$ & $29.4(6.0)$ & $28.4(5.7)$ & $28.3(5.7)$ \\
\hline$<30$ & 59 (4974) & $59(4868)$ & $66(6421)$ & $68(6579)$ \\
\hline \multicolumn{5}{|l|}{ Mean (SD) calcium intake ( $\mathrm{mg} /$ day): } \\
\hline Personal supplement & 0 & 0 & $582(538)$ & $582(520)$ \\
\hline Dietary intake & $804(489)$ & 798 (475) & $826(454)$ & $828(451)$ \\
\hline \multicolumn{5}{|l|}{ Mean (SD) blood pressure ( $\mathrm{mm} \mathrm{Hg}$ ): } \\
\hline Systolic & $126(17)$ & $126(17)$ & $125(17)$ & $125(17)$ \\
\hline Diastolic & $75(9)$ & $75(9)$ & $74(9)$ & $74(9)$ \\
\hline \multicolumn{5}{|l|}{ Medical history*: } \\
\hline HRT use (in trials or personal) & $49(4120)$ & $51(4199)$ & $54(5301)$ & $55(5401)$ \\
\hline $\begin{array}{l}\text { High serum cholesterol requiring } \\
\text { pills }\end{array}$ & $12(898)$ & $12(870)$ & $12(1110)$ & 12 (1094) \\
\hline Cardiovascular disease & 14 (1066) & 15 (1074) & 14 (1276) & 15 (1333) \\
\hline Hypertension & $34(2802)$ & 35 (2861) & $33(3144)$ & $32(3100)$ \\
\hline Stroke & $1.0(85)$ & $1.2(96)$ & $0.7(71)$ & $1.0(96)$ \\
\hline Myocardial infarction & $2.3(191)$ & $2.0(167)$ & $1.5(149)$ & $1.5(147)$ \\
\hline \multicolumn{5}{|l|}{ Smoking status*: } \\
\hline Never smoked & $52(4298)$ & $53(4307)$ & $52(5027)$ & $53(5121)$ \\
\hline Former smoker & $39(3260)$ & 38 (3154) & 41 (3995) & 41 (3979) \\
\hline Current smoker & $9(772)$ & $9(732)$ & $7(633)$ & $6(624)$ \\
\hline
\end{tabular}

$\mathrm{CaD}=$ allocation to calcium and vitamin $\mathrm{D}$ supplement. $\mathrm{HRT}=$ hormone replacement therapy.

*Data recorded at randomisation except for medical history and smoking status, which were recorded at entry to Women's Health Initiative clinical trials programme: $91 \%$ of participants in the CaD Study entered at their first annual visit for the WHI programme, and the remainder at their second annual visit.

\section{METHODS}

Analyses of WHI CaD Study

The design and results of the WHI CaD Study have been published in full. ${ }^{34}$ Medical records related to self reported medical events for myocardial infarction, stroke, and coronary revascularisation were adjudicated centrally by physician adjudicators using standardised definitions, and all deaths were also centrally adjudicated. ${ }^{4}$ We obtained the WHI limited access, clinical trials dataset from the National Heart Lung and Blood Institute. A statistical analysis proposal was submitted to the institute before the database was made available.

We attempted to replicate the approach of the WHI investigators where possible, carrying out prespecified analyses looking for interactions between pre-specified subgroups based on use (no use $v$ any use) and dose (0, 1-499, 500-999, $\geq 1000 \mathrm{mg}$ /day) of personal calcium supplements at randomisation for cardiovascular events. We pre-specified assessment of four cardiovascular end points and their combinations: myocardial infarction, coronary revascularisation (coronary artery bypass grafting or percutaneous coronary intervention), death from coronary heart disease, and stroke. Serial electrocardiograms were carried out in the WHI CaD Study, allowing detection of silent myocardial infarctions. Because silent myocardial infarctions were not determined in any of the trials in our meta-analysis, we analysed data separately for clinical myocardial infarctions and total myocardial infarctions (including clinical and silent myocardial infarction). We have reported four different composite end points: total deaths from myocardial infarction or coronary heart disease (the major outcome reported in the WHI CaD Study); clinical myocardial infarction or coronary revascularisation (representing clinical coronary heart disease events); clinical myocardial infarction or stroke (the composite end point most similar to that used in our meta-analysis of calcium monotherapy trials); and total myocardial infarction, coronary revascularisation, and death from coronary heart disease (representing all coronary heart disease events).

We have reported the baseline characteristics at the time of randomisation to the WHI CaD Study, whereas the WHI investigators reported these characteristics at the time of entry to the WHI programme. For body mass index and for dietary and supplemental calcium intake, we used the latest value recorded between screening and one month after randomisation to the study.

To assess the effect of calcium and vitamin $\mathrm{D}$ on the time to first event for each end point, we used Cox proportional hazards models stratified by age, prevalent cardiovascular disease at baseline, and randomisation status in the WHI Postmenopausal Hormone Therapy Trials and Dietary Modification Trial, following the approach of the WHI investigators. ${ }^{34}$ Comparisons between subgroups were assessed using interaction terms. The assumption of proportional hazards was tested by performing a test for 
proportionality of the interaction between variables included in the model and the logarithm of time.

\section{Meta-analysis of calcium with or without vitamin D}

Two recent systematic reviews have independently reviewed the effects of calcium and vitamin D supplements on vascular events. ${ }^{56}$ These reviews identified two trials of co-administered calcium and vitamin D with cardiovascular outcome data: the WHI CaD Study and a small, one year trial of 191 participants that reported 11 cardiovascular events during followup but did not provide specific details about these events. ${ }^{7}$ During the process of gathering data for our meta-analysis of calcium monotherapy, we obtained previously unpublished data from two studies that compared co-administered calcium and vitamin D with placebo. ${ }^{89}$ We therefore pooled data from these two studies together with the results for the WHI CaD Study participants who were not taking personal calcium supplements at randomisation in a meta-analysis of the effect of calcium and vitamin $\mathrm{D}$ on cardiovascular risk.

We then updated our previous meta-analysis of calcium supplements by including the results for the WHI CaD Study participants not taking personal calcium supplements at randomisation, to determine the effect of calcium with or without vitamin D on cardiovascular risk. Of note, only $1.2 \%$ of participants were taking non-protocol calcium supplements in the trials contributing patient-level data in our previous metaanalysis of calcium supplements, ${ }^{2}$ and two of the six studies contributing trial-level data permitted nonprotocol calcium supplements. ${ }^{910}$ We used the same methods as for our previous meta-analysis: ${ }^{2}$ the effect of calcium or calcium and vitamin D on the time to first event was assessed with Cox proportional hazards models stratified by study for patient-level data, and trial-level summary data were pooled using random effects models. ${ }^{2}$ The assumption of proportional hazards was tested as described above.
All analyses were performed using SAS version 9.1 (SAS Institute, Cary NC, USA) or Comprehensive Meta-analysis version 2 (Biostat, Englewood NJ, USA). All tests were two tailed and $\mathrm{P}<0.05$ was considered significant.

\section{RESULTS}

\section{Reanalysis of the WHI CaD Study}

Table 1 shows the characteristics of the participants at randomisation to the WHI CaD Study grouped by personal use of calcium supplements: $54 \%$ of participants were taking personal calcium supplements. The baseline characteristics of the participants allocated to calcium and vitamin D or to placebo seemed well matched for the subgroups defined by use of personal calcium supplement. However, participants using personal calcium supplements differed from those not using personal supplements in some factors associated with cardiovascular disease: age, body mass index, blood pressure, use of hormone replacement therapy, history of myocardial infarction or stroke, and smoking $(\mathrm{P}<0.05$ for these variables) (table 1$)$.

Table 2 shows the influence of personal use of calcium supplements on the effect of calcium and vitamin $\mathrm{D}$ on cardiovascular end points and mortality from all causes. There were significant interactions between allocation of calcium and vitamin $\mathrm{D}$ and personal use of calcium supplements for clinical myocardial infarction, stroke, and the composite end point of myocardial infarction or stroke. In women not taking personal calcium supplements, the hazard ratios with calcium and vitamin $\mathrm{D}$ were $1.16(\mathrm{P}=0.04)$ for the composite end point of clinical myocardial infarction or coronary revascularisation, $1.16(\mathrm{P}=0.05)$ for clinical myocardial infarction or stroke, $1.22(\mathrm{P}=0.05)$ for myocardial infarction, and 1.13-1.20 for the other cardiovascular end points. By contrast, in women taking personal calcium supplements, the hazard ratios for these end points with calcium and vitamin $\mathrm{D}$ were $0.83-1.08$.

Table 2 | Effect of allocation to calcium and vitamin D supplement on cardiovascular events among participants in the WHI CaD Study, grouped by personal use of calcium supplements at randomisation. Values are numbers (incidence per 1000 patient years) of events unless stated otherwise

\begin{tabular}{|c|c|c|c|c|c|c|c|c|c|}
\hline \multirow[b]{3}{*}{ Cardiovascular end point } & \multicolumn{4}{|c|}{ No personal use of calcium } & \multicolumn{4}{|c|}{ Any personal use of calcium } & \multirow[b]{3}{*}{$\begin{array}{l}\text { P value of } \\
\text { interaction }\end{array}$} \\
\hline & \multirow[b]{2}{*}{$\begin{array}{c}\mathrm{CaD} \\
(\mathrm{n}=8429)\end{array}$} & \multirow[b]{2}{*}{$\begin{array}{l}\text { Placebo } \\
(n=8289)\end{array}$} & \multicolumn{2}{|c|}{ Comparison } & \multirow[b]{2}{*}{$\begin{array}{c}\mathrm{CaD} \\
(\mathrm{n}=9747)\end{array}$} & \multirow[b]{2}{*}{$\begin{array}{c}\text { Placebo } \\
(n=9817)\end{array}$} & \multicolumn{2}{|c|}{ Comparison } & \\
\hline & & & $\begin{array}{c}\text { Hazard ratio } \\
(95 \% \mathrm{Cl})\end{array}$ & $P$ value & & & $\begin{array}{c}\text { Hazard ratio } \\
(95 \% \mathrm{Cl})\end{array}$ & $P$ value & \\
\hline Clinical MI & $209(3.5)$ & $168(2.9)$ & $1.22(1.00$ to 1.50$)$ & 0.05 & $180(2.7)$ & $196(2.9)$ & $0.92(0.75$ to 1.13$)$ & 0.4 & 0.04 \\
\hline Total MI† & $222(3.7)$ & $182(3.1)$ & $1.20(0.99$ to 1.47$)$ & 0.07 & $193(2.9)$ & $207(3.1)$ & $0.94(0.77$ to 1.14$)$ & 0.5 & 0.07 \\
\hline Revascularisation & $348(5.9)$ & $299(5.1)$ & $1.15(0.98$ to 1.34$)$ & 0.09 & $326(4.9)$ & $308(4.6)$ & 1.08 (0.93 to 1.27$)$ & 0.3 & 0.5 \\
\hline Stroke & $196(3.3)$ & $163(2.8)$ & $1.17(0.95$ to 1.44$)$ & 0.1 & $156(2.3)$ & $189(2.8)$ & 0.83 (0.67 to 1.02$)$ & 0.08 & 0.02 \\
\hline Total MI or CHD death & $268(4.5)$ & 229 (3.9) & 1.15 (0.97 to 1.38$)$ & 0.1 & $238(3.5)$ & $247(3.7)$ & $0.97(0.81$ to 1.16$)$ & 0.7 & 0.1 \\
\hline Clinical MI or revascularisation & $422(7.2)$ & $359(6.2)$ & $1.16(1.01$ to 1.34$)$ & 0.04 & $394(5.9)$ & $378(5.6)$ & $1.06(0.92$ to 1.23$)$ & 0.4 & 0.3 \\
\hline Clinical MI or stroke & $386(6.5)$ & $326(5.6)$ & $1.16(1.00$ to 1.35$)$ & 0.05 & $324(4.8)$ & $370(5.5)$ & 0.88 (0.76 to 1.02$)$ & 0.09 & 0.006 \\
\hline $\begin{array}{l}\text { Total MI, CHD death, or } \\
\text { revascularisation }\end{array}$ & $476(8.1)$ & $417(7.2)$ & $1.13(0.99$ to 1.29$)$ & 0.07 & $447(6.7)$ & $421(6.3)$ & 1.08 (0.94 to 1.23$)$ & 0.3 & 0.5 \\
\hline Death from all causes & $380(6.3)$ & $379(6.4)$ & $0.99(0.86$ to 1.14$)$ & 0.9 & $364(5.4)$ & $428(6.3)$ & $0.84(0.73$ to 0.97$)$ & 0.01 & 0.1 \\
\hline
\end{tabular}

$\mathrm{CaD}=$ allocation to calcium and vitamin $\mathrm{D}$ supplement. $\mathrm{Ml}=$ myocardial infarction. $\mathrm{CHD}=$ coronary heart disease.

*Interaction between $\mathrm{CaD}$ allocation and use or non-use of personal calcium supplements for each end point, testing the difference between subgroups.

†Includes clinically silent myocardial infarction diagnosed from changes in routine serial electrocardiograms. 
When the personal calcium supplement users were divided into three groups by daily supplement intake (1-499, 500-999, and $\geq 1000 \mathrm{mg} /$ day), there was no evidence of a relation between the dose of personal calcium supplements and the risk of cardiovascular events with randomisation to calcium and vitamin D for these end points (fig 1). There were no significant

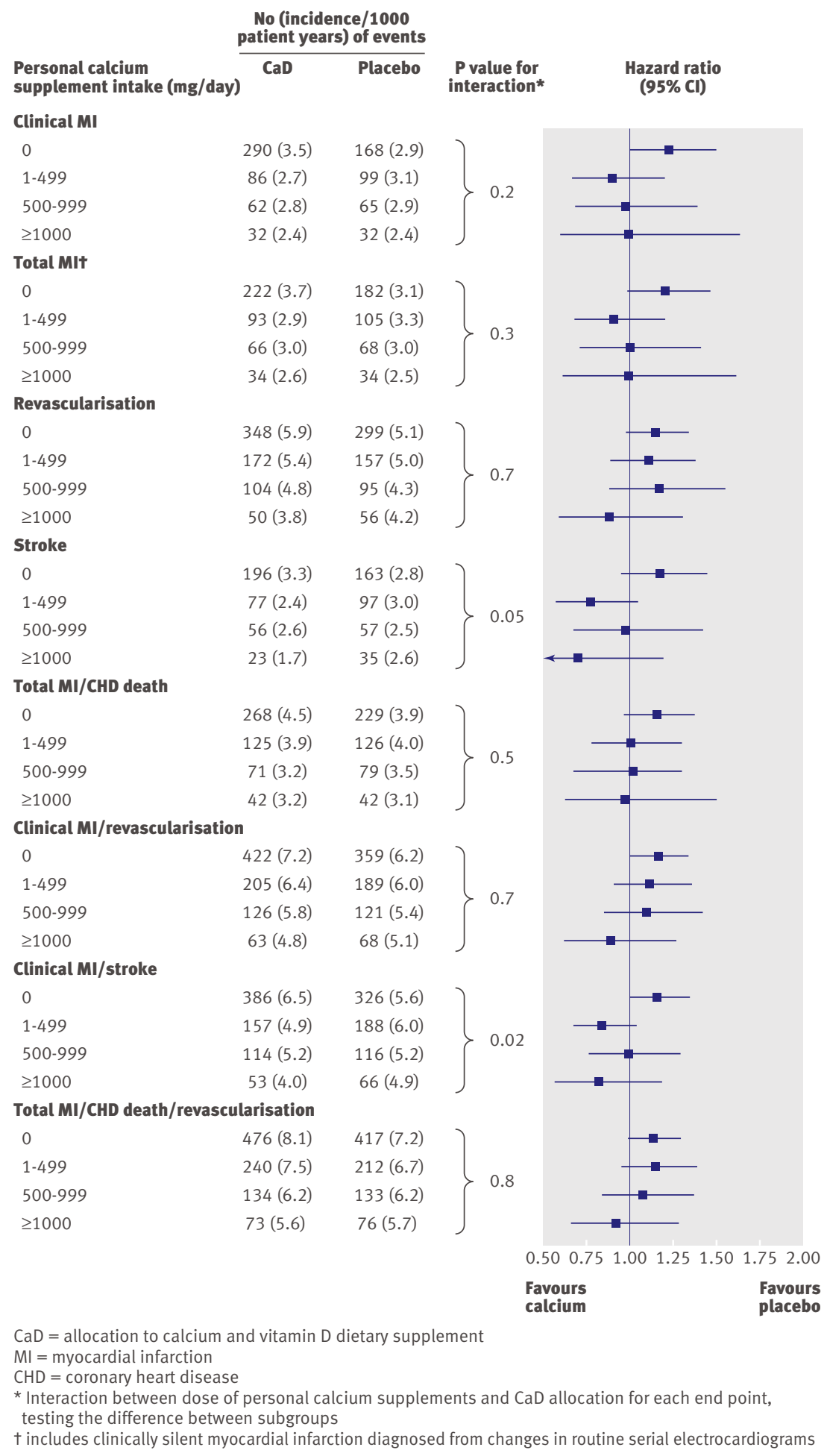

Fig 1 Influence of personal calcium supplement dose at randomisation on the effect of calcium and vitamin D on cardiovascular events in the WHI CaD Study three-way interactions between calcium and vitamin $\mathrm{D}$ allocation, personal calcium supplement use, and dietary calcium intake (above or below median) for cardiovascular end points $(\mathrm{P}>0.4)$. We repeated these analyses in the subgroup of women not using personal calcium supplements and found no interactions between calcium and vitamin $\mathrm{D}$ allocation and dietary calcium intake (above or below the median) for any cardiovascular end point $(\mathrm{P}>0.5)$. Finally, we repeated all these analyses using dietary calcium intake grouped by tertile, and the results were similar. Together, these analyses suggest that the relation between calcium and vitamin $\mathrm{D}$ allocation and cardiovascular events is independent of dietary calcium intake.

In a sensitivity analysis, we searched the WHI medication database to identify participants potentially taking calcium supplements not captured elsewhere, using WHI medication class codes for common calcium supplement preparations (791000, 791099, 781230, 783500, 783600, 483000, 489900, and 489910). We identified 930 participants listed as taking a calcium supplement before randomisation to calcium and vitamin $\mathrm{D}$ but recorded as having no supplemental intake of calcium at randomisation. When we repeated the analyses including these women classified as using personal calcium supplements, the results were similar (data not shown).

Personal vitamin D supplements were used in combination with personal calcium supplements by $44 \%$ of participants in the WHI database, and without personal calcium supplements by $3 \%$ of participants. When we repeated the analyses grouping participants by personal use of calcium and/or vitamin D supplements, the results were similar (data not shown).

Meta-analysis of calcium and vitamin D versus placebo We analysed trials comparing co-administered calcium and vitamin D with placebo. Complete triallevel data for cardiovascular events were available for 20090 participants from three trials - two trials with previously unpublished data gathered during preparation of our previous meta-analysis of calcium supplements, ${ }^{89}$ and data from the WHI CaD Study participants who were not taking personal calcium supplements at randomisation. In total, 465 individuals had an incident myocardial infarction, 477 an incident stroke, and 911 an incident myocardial infarction or stroke during an average trial duration weighted by study size of 6.2 years. Figure 2 shows that calcium and vitamin $\mathrm{D}$ significantly increased the risk of myocardial infarction (relative risk 1.21, $\mathrm{P}=0.04$ ), stroke (relative risk 1.20, $\mathrm{P}=0.05$ ), and the composite of myocardial infarction or stroke (relative risk 1.16, $\mathrm{P}=0.02$ ).

In a sensitivity analysis, we also included data from a study of calcium and vitamin D that only described cardiovascular event data in detail on trial withdrawals or deaths. ${ }^{7}$ The relative risks with calcium and vitamin $\mathrm{D}$ were 1.22 (95\% confidence interval 1.01 to 1.45 , $\mathrm{P}=0.03$ ) for myocardial infarction, 1.20 (1.00 to 1.43 , $\mathrm{P}=0.05)$ for stroke, and 1.17 (1.03 to $1.32, \mathrm{P}=0.02)$ for the composite end point. 


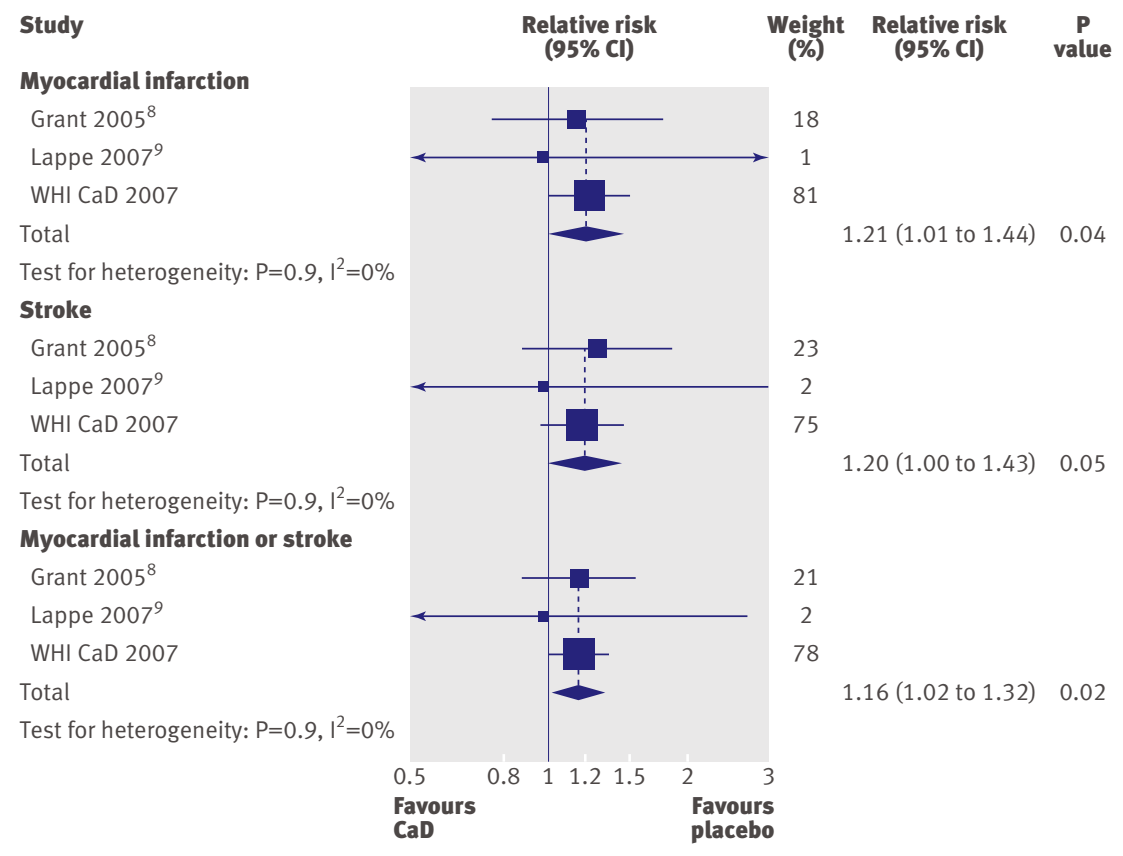

$\mathrm{CaD}=$ calcium and vitamin $\mathrm{D}$

Fig 2 Effect of calcium and vitamin D on cardiovascular events: based on trial-level data from two randomised, placebo controlled trials of calcium and vitamin $\mathrm{D}^{89}$ and the WHI CaD Study participants not taking personal calcium supplements at baseline. The first two trials included calcium monotherapy and calcium and vitamin D groups: we included data only from the calcium and vitamin $\mathrm{D}$ group compared with the placebo group

All cause mortality was available from three studies in which cardiovascular event data were available: Brazier et al, ${ }^{7}$ Grant et al for the RECORD trial, ${ }^{8}$ and the WHI CaD Study. In these studies, 1200 deaths occurred during follow-up, and the relative risk for death (all causes) with calcium and vitamin $\mathrm{D}$ was $1.01(0.90$ to $1.12, \mathrm{P}=0.9)$.

Meta-analysis of calcium with or without vitamin D versus placebo

As the magnitude of the risk of cardiovascular events with calcium and vitamin D was similar to that observed with calcium alone, we updated our previous meta-analysis by incorporating the results for the WHI $\mathrm{CaD}$ participants who were not taking personal calcium supplements at randomisation, and the data from the calcium and vitamin $\mathrm{D}$ arm of the Lappe study; ${ }^{9}$ this arm was not included in our previous meta-analysis.

\section{Patient-level data}

Patient-level data were available for 24869 people in six trials (WHI CaD and five randomised, placebo controlled trials of calcium supplements). ${ }^{111-15}$ In total, 631 individuals had an incident myocardial infarction, 669 an incident stroke, 1248 an incident myocardial infarction or stroke, and 1765 died during a mean follow-up of 5.9 years. Figure 3 shows that calcium or calcium and vitamin $\mathrm{D}$ supplements increased the risk of myocardial infarction (hazard ratio 1.26, $\mathrm{P}=0.005)$, stroke (1.19, $\mathrm{P}=0.03)$, and the composite end point of myocardial infarction or stroke (1.17, $\mathrm{P}=0.005$ ). The hazard ratio for death (all causes) was 1.04 (0.95 to $1.14, \mathrm{P}=0.4$ ).

The number needed to treat with calcium or calcium and vitamin $\mathrm{D}$ for five years to cause one incident event was 240 for myocardial infarction, 283 for stroke, and 178 for the composite end point. The corresponding number needed to treat to prevent one fracture was 302. Treating 1000 people with calcium or calcium and vitamin $\mathrm{D}$ for five years would cause an additional six myocardial infarctions or strokes and prevent three fractures.

\section{Trial-level data}

Complete trial-level data were available for 28072 participants in nine trials (the WHI CaD study and eight randomised, placebo controlled trials of calcium supplements). ${ }^{18911-17}$ In total, 676 individuals had an incident myocardial infarction, 764 an incident stroke, 1384 an incident myocardial infarction or stroke, and 1835 died during an average trial duration weighted by study size of 5.7 years. Figure 4 shows that the results were similar to the patient-level analyses. Calcium or calcium and vitamin D supplements increased the risk of myocardial infarction (relative risk 1.24, $\mathrm{P}=0.004$ ) and the composite of myocardial infarction/stroke (1.15, $\mathrm{P}=0.009)$. The relative risk of death (all causes) was 1.04 (0.95 to $1.13, \mathrm{P}=0.5$ ).

In a sensitivity analysis, we added all available data from three further randomised, placebo controlled trials of calcium supplements ${ }^{1018}{ }^{19}$ and one trial of calcium and vitamin D supplements ${ }^{7}$ (limited to data on deaths in trial or trial withdrawals) to the analysis of complete trial-level data. Data were available for 29277 participants (WHI CaD Study, 11 trials of calcium supplements, and one trial of calcium and vitamin D): 679 individuals had an incident myocardial infarction, 768 an incident stroke, 1393 an incident myocardial infarction or stroke, and 1857 died during follow-up. With calcium or calcium and vitamin D supplements, the relative risks were 1.25 (1.08 to 1.45 , $\mathrm{P}=0.003$ ) for myocardial infarction, 1.15 (1.00 to 1.32 , $\mathrm{P}=0.06$ ) for stroke, 1.15 (1.04 to $1.27, \mathrm{P}=0.008$ ) for the composite of myocardial infarction or stroke, and 1.04 $(0.95$ to $1.13, \mathrm{P}=0.4)$ for death.

\section{DISCUSSION}

In the Women's Health Initiative Calcium/Vitamin D Supplementation (WHI CaD) Study personal use of calcium supplement at randomisation significantly influenced the effect of randomisation to calcium and vitamin $\mathrm{D}$ on the risk of cardiovascular events. In the entire WHI cohort, there were significant interactions between calcium and vitamin $\mathrm{D}$ and personal calcium supplement use for myocardial infarction and for stroke. In the $46 \%$ of the WHI CaD participants who were not taking personal calcium supplements at randomisation, the hazard ratios for cardiovascular events with calcium and vitamin D ranged from 1.13 to 1.22 . By contrast, in the participants taking personal calcium supplements at randomisation, allocation to calcium 
and vitamin D did not alter cardiovascular risk. In women not taking personal calcium supplements at randomisation, the hazard ratios for clinical myocardial infarction (1.22) and stroke (1.17) were similar to those observed in our meta-analysis of trials of calcium monotherapy (1.31 and 1.20 , respectively). ${ }^{2}$

By itself, this analysis of the WHI CaD Study data does not provide definitive evidence of an adverse effect of calcium and vitamin D on cardiovascular events. However, when these data are pooled with previously unpublished data from two other placebo controlled trials of calcium and vitamin $\mathrm{D}$, there are consistent increases in the risk of myocardial infarction and stroke that are statistically significant and are of similar size to the risks observed with calcium supplements used without vitamin D. Further, when the results for calcium and vitamin $\mathrm{D}$ are taken together with those from trials of calcium used as monotherapy, they provide consistent evidence from 13 randomised, placebo controlled trials involving about 29000 participants with about 1400 incident myocardial infarctions and strokes that calcium supplements with or without vitamin $\mathrm{D}$ increase the risk of cardiovascular events.

The size of this increase is modest, about 25\%-30\% for myocardial infarction and 15\%-20\% for stroke, but, because of the widespread use of calcium supplements either alone or with vitamin $\mathrm{D}$, even small increases in cardiovascular disease incidence may translate to a substantial population burden of disease, particularly in older age groups. Furthermore, comparisons of the benefits of calcium on fracture prevention with the risk of cardiovascular events suggest that the risk to benefit profile is unfavourable: in our analysis, treating 1000 patients with calcium or calcium and vitamin $\mathrm{D}$ for five years would cause an additional six myocardial infarctions or strokes (number needed to harm of 178) and prevent only three fractures (number needed to treat of 302).

\section{Limitations of study}

The current analysis has some limitations. We used the publicly accessible, limited access dataset of the WHI clinical trials for these analyses, so the analysis is limited to the information available in this dataset. Several vascular end points are potentially evaluable; we prespecified those which allowed the most accurate comparisons with previous analyses. Subgroup analysis raises several issues, including false positive results and over-interpretation of findings. ${ }^{2021}$ To minimise these risks, we pre-specified the variable of interest (personal calcium supplement use) for this analysis before obtaining the WHI dataset, assessed its effect using interaction tests, and followed recommended approaches for subgroup analysis and inter pretation. ${ }^{2122}$ The hypothesis that the use of personal calcium supplements might interact with the calcium and vitamin D treatment effect in the WHI CaD Study was based on plausibility from our finding in trials of calcium monotherapy, and deviation from normal clinical trial practice (allowing trial participants free access to the intervention being studied) is unusual and has the potential to mask both adverse and beneficial effects.

We followed the approach of the WHI authors in not adjusting $\mathrm{P}$ values for multiple subgroup analyses, and instead estimated the likelihood of false positive tests, ${ }^{3}$ an approved approach for addressing multiplicity of

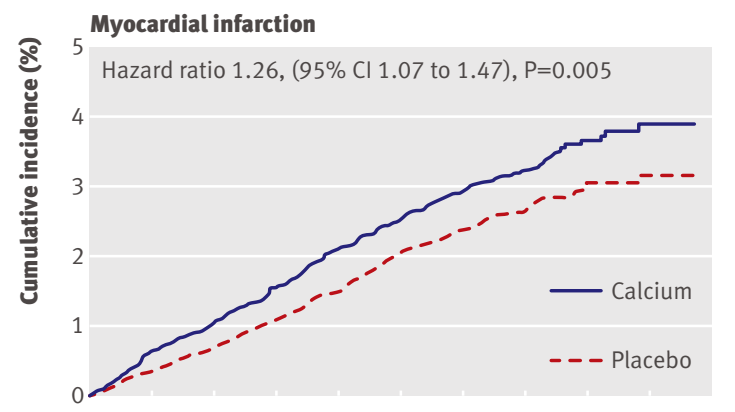

\section{No at risk}
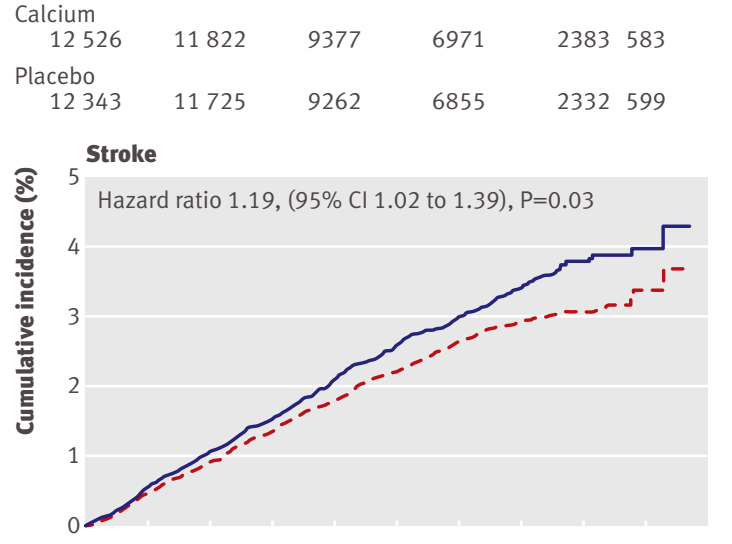

No at risk

Calcium

$\begin{array}{llllll}12526 & 11817 & 9391 & 6982 & 2383 & 585\end{array}$ Placebo

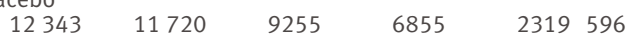

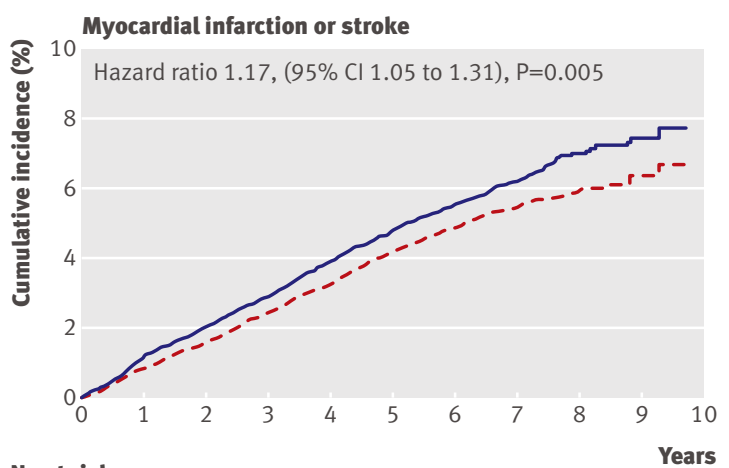

No at risk

Calcium

$$
\begin{array}{lllll}
12526 & 11746 & 9281 & 6870 & 2334 \\
\hline
\end{array}
$$

Placebo

12343

11660

9167

6761

2287586

Fig 3 | Effect of calcium supplements with or without vitamin D on cardiovascular events: based on patient-level data. The panels show the time to first event for 24869 participants in five trials of calcium supplements, ${ }^{1811-15}$ and the WHI CaD Study participants not taking personal calcium supplements at baseline 


\section{Study}

Myocardial infarction

Baron $1999^{13}$

Grant $2005^{8}$

Grant 2005 Vit D ${ }^{8}$

Prince $2006^{16}$

Reid $2006^{114}$

Lappe $2007^{9}$

WHI CaD 2007

Reid $2008^{15}$

Total

Test for heterogeneity: $P>0.9,\left.\right|^{2}=0 \%$

\section{Stroke}

Reid $1993^{1112}$

Baron $1999^{13}$

Grant $2005^{8}$

Grant 2005 Vit D 8

Prince $2006^{16}$

Reid $2006^{114}$

Bonnick $2007^{17}$

Lappe $2007^{9}$

WHI CaD 2007

Total

Test for heterogeneity: $P>0.9, I^{2}=0 \%$

Myocardial infarction or stroke

Reid $1993^{11} 12$

Baron $1999^{13}$

Grant $2005^{8}$

Grant 2005 Vit D ${ }^{8}$

Prince $2006^{16}$

Reid $2006^{114}$

Bonnick $2007^{17}$

Lappe $2007^{9}$

WHI CaD 2007

Reid $2008^{15}$

Total

Test for heterogeneity: $\mathrm{P}>0.9, \mathrm{I}^{2}=0 \%$

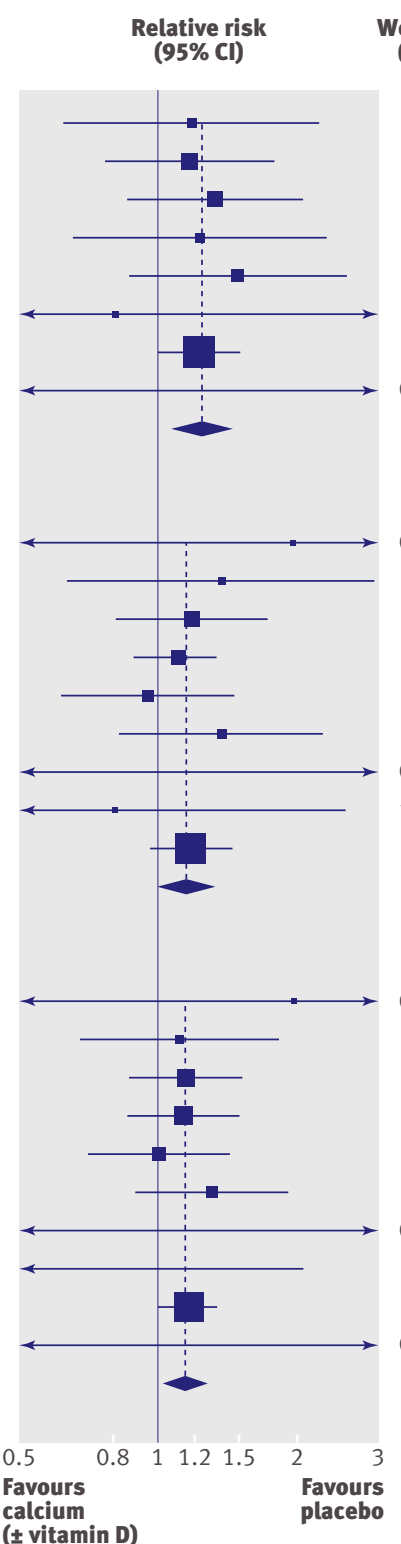

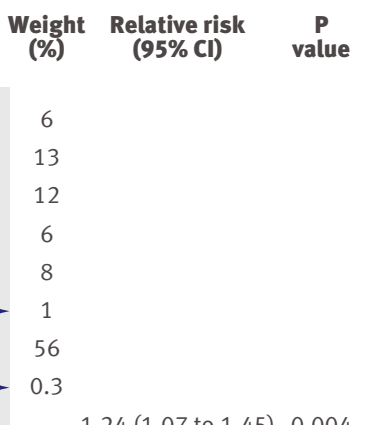

1.24 (1.07 to 1.45$) \quad 0.004$

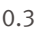

3

14

16

11

8

0.3

1.5

47

1.15 (1.00 to 1.32$) \quad 0.06$

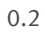

4

14

14

8

7

0.2

51

0.1

1.15 (1.03 to 1.27$) \quad 0.009$ personal calcium differed from women not using personal calcium in a number of factors that might influence cardiovascular outcomes. However, the WHI investigators have previously reported no significant interactions with these factors, calcium and vita$\min \mathrm{D}$, and risk of death from myocardial infarction or coronary heart disease - except for body mass index, with obese women having a lower risk of death from myocardial infarction or coronary heart disease with calcium and vitamin $\mathrm{D}$ than non-obese women. ${ }^{4}$ Obesity does not explain our findings since it was more common in women not using personal calcium, and would have tended to obscure an interaction effect.

In the WHI $\mathrm{CaD}$ participants the incidence of cardiovascular events was relatively low, reflecting the comparative youth of the cohort. Thus, despite its size and long duration, the WHI CaD Study had insufficient power to detect small effect sizes, particularly when subgroups are considered. For example, in women not taking personal calcium supplements at randomisation, the study had $80 \%$ power to detect a $33 \%$ increase in clinical myocardial infarction.

The WHI CaD Study accounts for 75\%-80\% of the weighting in the meta-analyses of co-administered calcium and vitamin $\mathrm{D}$, and $45 \%-55 \%$ of the weighting in the meta-analysis of calcium with or without vitamin D. However, the results for the individual studies in all the meta-analyses are quite consistent and do not suggest an undue influence of a single outlying study or WHI CaD.

\section{Interpretation of results}

For most of its participants, the WHI CaD Study assessed the impact of adding calcium and vitamin D to personal calcium supplements, effectively comparing a higher dose of calcium with a lower dose of calcium. By restricting the analyses to women not taking personal calcium supplements, we were able to estimate the effect of calcium and vitamin D on cardiovascular events, observing increased risks of these events with calcium and vitamin D. In women taking personal calcium supplements at randomisation, the addition of calcium and vitamin $\mathrm{D}$ did not increase cardiovascular risk, and the risk of cardiovascular events with calcium and vitamin D was also not affected by the dose of personal calcium supplements. This suggests that there may not be a dose-response relationship between calcium supplements and the risk of cardiovascular events. Thus, even doses of $<500 \mathrm{mg}$ /day might be associated with an increased risk of cardiovascular events similar to doses $\geq 1000 \mathrm{mg} /$ day. This would be consistent with the notion that the abrupt change in plasma calcium concentration after supplement ingestion causes the adverse effect, rather than it being related to the total calcium load ingested..$^{2425}$

In the entire WHI cohort there was no significant interaction between calcium and vitamin $\mathrm{D}$, personal calcium supplement use, and mortality — and therefore no evidence of a difference in mortality risk with calcium and vitamin $\mathrm{D}$ in the subgroups defined by 


\section{WHAT IS ALREADY KNOWN ON THIS TOPIC}

A recent meta-analysis suggested that calcium supplements taken without vitamin D

increase the risk of myocardial infarction

The Women's Health Initiative reported no effect of calcium and vitamin D supplements on cardiovascular events, but most of the participants were taking personal, non-protocol calcium supplements at study entry

\section{WHAT THIS STUDY ADDS}

Re-analysis of the Women's Health Initiative data shows women allocated to calcium and vitamin D administration who were not taking personal calcium supplements were at increased risk of cardiovascular events

Meta-analyses of trials involving 29000 people found that calcium supplements used with or without vitamin D modestly increase cardiovascular risk, suggesting their use in osteoporosis management should be reassessed personal calcium use. In women not taking personal calcium supplements, no increase in mortality was observed with calcium and vitamin $\mathrm{D}$ despite the increased risk of cardiovascular events. The most likely explanation is that participants in WHI were at low risk of cardiovascular events and death. A 15\% increase in myocardial infarction and stroke would lead to only a $1 \%-3 \%$ increase in total mortality if $10 \%-20 \%$ of the additional events led to death during follow-up. The subgroup analysis did not have sufficient power to detect such a difference, but the $95 \%$ confidence intervals for mortality ( 0.86 to 1.14 ) encompass an effect of this size.

If calcium supplements do increase cardiovascular risk it is important to consider the potential underlying mechanisms. ${ }^{25}$ Calcium supplements acutely increase serum calcium concentration by a modest amount, ${ }^{24}$ an effect that is sustained during long term treatment, as evidenced by lower levels of parathyroid hormone..$^{15}$ Serum calcium concentrations are positively associated with carotid artery plaque thickness ${ }^{26}$ aortic calcification, ${ }^{27}$ incidence of myocardial infarction, ${ }^{28-30}$ and mortality. ${ }^{31}$ These findings are consistent with observational data suggesting increased risk of cardiovascular events and death in primary hyperparathyroidism, a condition in which serum calcium concentration is elevated. ${ }^{3233}$

The process of vascular calcification is a complex, regulated process similar to osteogenesis. ${ }^{34}$ It is possible that the increase in serum calcium concentrations from calcium supplements influences vascular calcification by altering regulators of calcification such as fetuin A, pyrophosphate, and bone morphogenic protein-7, or by directly binding to the calcium-sensing receptor that is expressed on vascular smooth muscle cells. ${ }^{25}$ Exposing cultures of vascular smooth muscle cells to increased concentrations of calcium results in increased mineralisation of the cultures. ${ }^{35}$ Supporting this hypothesis are studies of patients with renal impairment, in whom calcium supplements accelerate vascular calcification and increase mortality in both dialysis and pre-dialysis populations. ${ }^{36-38}$

It is also possible that calcium supplements adversely affect risk of arterial thrombus formation.
Acute hypercalcaemia in rats increases blood coaguability, ${ }^{39}$ potentially via an effect on platelets since calcium-sensing receptors are found on these cells. ${ }^{40}$

Therefore, extracellular calcium concentrations might affect the function of several cells that are implicated in the pathogenesis of vascular events. All these possibilities require further evaluation.

\section{Conclusions}

Calcium and vitamin D supplements increased the risk of cardiovascular events in the WHI CaD participants who were not taking personal calcium supplements at the time of randomisation. When these results are taken together with the results of other clinical trials of calcium supplements, with or without vitamin D, they strongly suggest that calcium supplements modestly increase the risk of cardiovascular events, particularly myocardial infarction. These data justify a reassessment of the use of calcium supplements in older people.

The Women's Health Initiative is conducted and supported by the National Heart, Lung and Blood Institute (NHLBI) in collaboration with the WHI Study Investigators. This manuscript was prepared using a limited access dataset obtained from the NHLBI and does not necessarily reflect the opinions or views of the WHI or the NHLBI.

We thank Professor John Baron who provided patient-level data on cardiovascular events from his study, Professor Joan Lappe who provided trial-level data on her study, and Graeme MacLennan from the RECORD trial

Contributors: MJB, AG, and IRR drafted the study protocol. All authors provided individual patient data from their studies. MJB and GDG performed the analyses. MJB drafted the paper. All authors critically reviewed the paper. MIB had full access to all the data in the study and takes responsibility for the integrity of the data and the accuracy of the data analysis. MJB is the guarantor of the paper

Funding: Funded by the Health Research Council of New Zealand, and the University of Auckland School of Medicine Foundation. AA is funded by a Career Scientist award of the Chief Scientist Office of the Scottish

Government Health Directorates. The Health Services Research Unit is funded by the Chief Scientist Office of the Scottish Government Health Directorates

The study sponsors had no role in design and conduct of the study: collection, management, analysis, and interpretation of the data; or preparation, review, and approval of the manuscript. The authors are independent from the funders.

Competing interests: All authors have completed the Unified Competing Interest form at www.icmje.org/coi_disclosure.pdf (available on request from the corresponding author) and declare that no author has support from companies for the submitted work; IRR has received research support from and acted as a consultant for Fonterra and had study medications for clinical trials of calcium supplementation supplied by Mission Pharmacal, and AA had study medications for clinical trials of calcium supplementation supplied by Shire Pharmaceuticals and Nycomed; no other relationships or activities that could appear to have influenced the submitted work.

Ethical approval: Not required.

Data sharing: No additional data available.

1 Bolland MJ, Barber PA, Doughty RN, Mason B, Horne A, Ames R, et al. Vascular events in healthy older women receiving calcium supplementation: randomised controlled trial. BM/2008;336:262-6.

2 Bolland MJ, Avenell A, Baron JA, Grey A, MacLennan GS, Gamble GD, et al. Effect of calcium supplements on risk of myocardial infarction and cardiovascular events: meta-analysis. BMJ 2010;341:C3691.

3 Jackson RD, LaCroix AZ, Gass M, Wallace RB, Robbins I, Lewis CE, et al. Calcium plus vitamin $D$ supplementation and the risk of fractures. N Engl I Med 2006;354:669-83.

4 Hsia J, Heiss G, Ren H, Allison M, Dolan NC, Greenland P, et al. Calcium/vitamin D supplementation and cardiovascular events. Circulation 2007;115:846-54 
5 Chung M, Balk EM, Brendel M, Ip S, Lau J, Lee J, et al. Vitamin D and calcium: a systematic review of health outcomes. Evidence report no 183. (Prepared by the Tufts Evidence-based Practice Center unde contract no HHSA 290-2007-10055-I.) AHRQ publication no 09E015. Agency for Healthcare Research and Quality, 2009.

6 Wang L, Manson JE, Song Y, Sesso HD. Systematic review: vitamin D and calcium supplementation in prevention of cardiovascular events. Ann Intern Med 2010;152:315-23.

7 Brazier M, Grados F, Kamel S, Mathieu M, Morel A, Maamer M, et al. Clinical and laboratory safety of one year's use of a combination calcium + vitamin D tablet in ambulatory elderly women with vitamin D insufficiency: results of a multicenter, randomized, double-blind, placebo-controlled study. Clin Ther 2005;27:1885-93.

8 Grant AM, Avenell A, Campbell MK, McDonald AM, MacLennan GS, McPherson GC, et al. Oral vitamin D3 and calcium for secondary prevention of low-trauma fractures in elderly people (Randomised Evaluation of (alcium Or vitamin D: RECORD): a randomised placebo controlled trial. Lancet 2005;365:1621-8.

9 Lappe JM, Travers-Gustafson D, Davies KM, Recker RR, Heaney RP. Vitamin $D$ and calcium supplementation reduces cancer risk: results of a randomized trial. Am J Clin Nutr 2007;85:1586-91.

10 Riggs BL, O’Fallon WM, Muhs J, O’Connor MK, Kumar R, Melton LJ 3rd. Long-term effects of calcium supplementation on serum parathyroid hormone level, bone turnover, and bone loss in elderly women. J Bone Miner Res 1998;13:168-74.

11 Reid IR, Ames RW, Evans MC, Gamble GD, Sharpe SJ. Effect of calcium supplementation on bone loss in postmenopausal women. $N$ Engl Med 1993;328:460-4.

12 Reid IR, Ames RW, Evans MC, Gamble GD, Sharpe SJ. Long-term effects of calcium supplementation on bone loss and fractures in postmenopausal women: a randomized controlled trial. Am J Med 1995;98:331-5.

13 Baron JA, Beach M, Mandel JS, van Stolk RU, Haile RW, Sandler RS, et al. Calcium supplements for the prevention of colorectal adenomas. Calcium Polyp Prevention Study Group. N Engl J Med 1999;340:101-7.

14 Reid IR, Mason B, Horne A, Ames R, Reid HE, Bava U, et al. Randomized controlled trial of calcium in healthy older women. $A m$ Med 2006;119:777-85

15 Reid IR, Ames R, Mason B, Reid HE, Bacon Cl, Bolland MJ, et al. Randomized controlled trial of calcium supplementation in healthy, non-osteoporotic, older men. Arch Intern Med 2008;168:2276-82.

16 Prince RL, Devine A, Dhaliwal SS, Dick IM. Effects of calcium supplementation on clinical fracture and bone structure: results of a 5-year, double-blind, placebo-controlled trial in elderly women. Arch Intern Med 2006;166:869-75.

17 Bonnick S, Broy S, Kaiser F, Teutsch C, Rosenberg E, DeLucca P, et al. Treatment with alendronate plus calcium, alendronate alone, or calcium alone for postmenopausal low bone mineral density. Curr Med Res Opin 2007;23:1341-9.

18 Dawson-Hughes B, Dallal GE, Krall EA, Sadowski L, Sahyoun N, Tannenbaum S. A controlled trial of the effect of calcium supplementation on bone density in postmenopausal women. $N$ Engl J Med 1990;323:878-83.

19 Bonithon-Kopp C, Kronborg O, Giacosa A, Rath U, Faivre J. Calcium and fibre supplementation in prevention of colorectal adenoma recurrence: a randomised intervention trial. European Cancer Prevention Organisation Study Group. Lancet 2000;356:1300-6.

20 Yusuf S, Wittes J, Probstfield J, Tyroler HA. Analysis and interpretation of treatment effects in subgroups of patients in randomized clinical trials. JAMA 1991;266:93-8.

21 Assmann SF, Pocock SJ, Enos LE, Kasten LE. Subgroup analysis and other (mis)uses of baseline data in clinical trials. Lancet 2000;355:1064-9.
22 Lagakos SW. The challenge of subgroup analyses-reporting without distorting. N Engl J Med 2006;354:1667-9.

23 Wang R, Lagakos SW, Ware JH, Hunter DJ, Drazen JM. Statistics in medicine-reporting of subgroup analyses in clinical trials. $N$ Engl J Med 2007;357:2189-94.

24 Reid IR, Schooler BA, Hannan SF, Ibbertson HK. The acute biochemical effects of four proprietary calcium preparations. Aust NZ J Med 1986;16:193-7.

25 Reid IR, Bolland MJ, Grey A. Does calcium supplementation increase cardiovascular risk? Clin Endocrinol (Oxf) 2010;73:689-95.

26 Rubin MR, Rundek T, McMahon DJ, Lee HS, Sacco RL, Silverberg SJ. Carotid artery plaque thickness is associated with increased serum calcium levels: the Northern Manhattan study. Atherosclerosis 2007;194:426-32

27 Bolland MJ, Wang TK, van Pelt NC, Horne AM, Mason BH, Ames RW, et al. Abdominal aortic calcification on vertebral morphometry images predicts incident myocardial infarction. J Bone Miner Res 2010;25:505-12.

28 Lind L, Skarfors E, Berglund L, Lithell H, Ljunghall S. Serum calcium: a new, independent, prospective risk factor for myocardial infarction in middle-aged men followed for 18 years. J Clin Epidemiol 1997;50:967-73.

29 Jorde R, Sundsfjord J, Fitzgerald P, Bonaa KH. Serum calcium and cardiovascular risk factors and diseases: the Tromso study. Hypertension 1999;34:484-90.

30 Foley RN, Collins AJ, Ishani A, Kalra PA. Calcium-phosphate levels and cardiovascular disease in community-dwelling adults: the Atherosclerosis Risk in Communities (ARIC) Study. Am Heart J 2008;156:556-63.

31 Leifsson BG, Ahren B. Serum calcium and survival in a large health screening program. J Clin Endocrinol Metab 1996;81:2149-53.

32 Nilsson IL, Yin L, Lundgren E, Rastad J, Ekbom A. Clinical presentation of primary hyperparathyroidism in Europe-nationwide cohort analysis on mortality from nonmalignant causes. J Bone Miner Res 2002;17(suppl 2):N68-74.

33 Vestergaard P, Mollerup CL, Frokjaer VG, Christiansen P, Blichert-Toft M, Mosekilde L. Cardiovascular events before and after surgery for primary hyperparathyroidism. World J Surg 2003;27:216-22.

34 Demer LL. A skeleton in the atherosclerosis closet. Circulation 1995;92:2029-32.

35 Alam MU, Kirton JP, Wilkinson FL, Towers E, Sinha S, Rouhi M, et al. Calcification is associated with loss of functional calcium-sensing receptor in vascular smooth muscle cells. Cardiovasc Res 2009;81:260-8.

36 Goodman WG, Goldin J, Kuizon BD, Yoon C, Gales B, Sider D, et al. Coronary-artery calcification in young adults with end-stage renal disease who are undergoing dialysis. $N$ Engl J Med 2000;342:1478-83.

37 Block GA, Raggi P, Bellasi A, Kooienga L, Spiegel DM. Mortality effect of coronary calcification and phosphate binder choice in incident hemodialysis patients. Kidney Int 2007;71:438-41.

38 Russo D, Miranda I, Ruocco C, Battaglia Y, Buonanno E, Manzi S, et al. The progression of coronary artery calcification in predialysis patients on calcium carbonate or sevelamer. Kidney Int 2007;72:1255-61.

39 Hilgard P. Experimental hypercalcaemia and whole blood clotting. Clin Path 1973;28:616-9.

40 House MG, Kohlmeier L, Chattopadhyay N, Kifor O, Yamaguchi T, Leboff MS, et al. Expression of an extracellular calcium-sensing receptor in human and mouse bone marrow cells. J Bone Miner Res 1997;12:1959-70.

Accepted: 18 February 2011 